\title{
Brain Activity during Stereognostic Discrimination Using the Tongue Measured By Functional Near-Infrared Spectroscopy
}

\author{
Shigenori Kawagishi ${ }^{{ }^{*}}$, Toshiko Tanaka ${ }^{2}$, Kenichi Yoshino ${ }^{3}$ and Megumi Shimodozono 4 \\ ${ }^{1}$ Department of Health Improvement, Kyushu Dental University, Kitakyushu, Japan \\ ${ }^{2}$ Department of Physical Functions, Kyushu Dental University, Kitakyushu, Japan \\ ${ }^{3}$ Department of Oral Health Management, School of Oral Health Sciences, Kyushu Dental University, Kitakyushu, Japan \\ ${ }^{4}$ Department of Rehabilitation and Physical Medicine, Graduate School of Medical and Dental Sciences, Kagoshima University, Kagoshima, Japan
}

"Corresponding author: Shigenori Kawagishi, Department of Health Improvement, Kyushu Dental University, 2-6-1 Manazuru, Kokurakita-ku, Kitakyushu 803-8580, Japan, Tel: (+81)-93-285-3039; Fax: (+81)-93-582-6000; E-mail: kawagisi@kyu-dent.ac.jp

Rec date: Aug 27, 2014, Acc date: Sep 22, 2014, Pub date: Sep 29, 2014

Copyright: (C) 2014 Kawagishi S, et al. This is an open-access article distributed under the terms of the Creative Commons Attribution License, which permits unrestricted use, distribution, and reproduction in any medium, provided the original author and source are credited.

\begin{abstract}
We previously reported our investigation of the stereognostic ability of the tongue to recognize the shape and size of materials in the mouth and its clinical applications. In the present study, we observed brain activity while performing stereognostic discrimination using the tongue; the changes in levels of oxyhemoglobin (oxy-Hb), deoxyhemoglobin (deoxy-Hb), and total hemoglobin (total- $\mathrm{Hb}$ ) in the frontal cortex area of 37 healthy individuals (18-75 years old) were measured using a functional near-infrared spectrometer with 45 channels. As an experimental task, the stereognostic test piece (TP) was placed on the center of the tongue dorsum, and subjects moved the TP for shape discrimination without letting it touch the teeth or gums. As a control task, subjects did not move the TP placed on the tongue. Stereognostic task-attributed changes in Hb levels were obtained by subtracting the $\mathrm{Hb}$ level of the control task from that of the experimental task. During the stereognostic task, oxy- $\mathrm{Hb}$ levels increased in the frontal cortex, including the prefrontal area, in most subjects. However, the results also demonstrated large individual variability. When the degree of change in $\mathrm{Hb}$ levels during the stereognostic task was mapped, it was found that the changes in the oxy- and total-Hb levels were similar, while the deoxy- $\mathrm{Hb}$ level did not change to the same degree. When subjects were divided into three groups, highly-, moderately- and mildlyincreased oxy- $\mathrm{Hb}$ levels, there was a significant negative correlation between age and degree of oxy- $\mathrm{Hb}$ level increase. The present study suggests stereognosis may be involved in several different information processing pathways, and that aging may also be a factor in the large variability we observed.
\end{abstract}

Keywords: Oral stereognosis; Tongue; Test pieces; Brain activity; fNIRS; Aging

\section{Introduction}

In our aging society, the increase of the elderly population and the accompanying increase of patients with cerebrovascular disorders have led to a rise in the prevalence of eating and swallowing dysfunction. Because the rehabilitation of proper eating and swallowing is important, the motor function of the tongue has been highly studied due to its role in these processes [1-3]. By examining stroke patients with dysphagia using video fluorography, Han et al. [1,2] studied the factors influencing the functional prognosis of aspiration and swallowing and reported that the tongue played an essential role in these functions.

The importance of sensory feedback in motor control and learning is well known [4]. The size and shape of an intraoral bolus of food provides oral sensory information important for the various stages of eating and swallowing, such as the masticatory, oral, and pharyngeal stages [5], and the tongue is responsible for oral stereognosis [6-11]. However, it is not clear to what degree impairment of higher sensory perception of the tongue, including stereognosis, is associated with disorders of tongue movement and deglutition.

We previously developed an objective evaluation method of oral stereognostic ability, using twenty types of test pieces, and a rehabilitation method that targets recovery of this ability [12]. Furthermore, we showed that oral stereognosis is achieved mainly through sensory information from the tongue and that this ability declines with age. Further study revealed that it is possible to screen for a decline in stereognostic ability in the elderly with the use of six types of test pieces [13]. In addition, we preliminarily reported that oral stereognostic ability in poststroke patients with dysphagia had a significant positive correlation with a video fluoroscopic dysphagia scale, especially in the oral phase [14].

Focus on brain areas that regulate the higher-order sensory and motor functions of the tongue is important to elucidate the relationship between the decrease in oral sensory, tongue motor disability, and dysphagia. Functional brain imaging studies using Functional Magnetic Resonance Imaging (fMRI) have reported that brain activity is associated with oral functions such as mastication [15-18]; however, there have been few reports about the brain activity in relation to oral stereognosis. In the present study, we investigated the changes in hemoglobin $(\mathrm{Hb})$ levels in various age groups using Functional Near-Infrared Spectroscopy (fNIRS) to determine both the brain activity of individuals performing a test piece shape discrimination task, and the effect of aging on this activity. 


\section{Materials and Methods}

\section{Subjects}

The subjects were 37 healthy volunteers ( 30 men, 7 women aged 18-75 years) without masticatory or swallowing dysfunction. Approval for the study protocol was obtained from the ethics committee of Kyushu Dental University. Written consent was obtained from all subjects following sufficient explanation of the purpose and content of the research.

\section{Measurement procedure of hemoglobin levels during the oral stereognostic tasks}

Three polyethylene test pieces (Figure 1, $13 \mathrm{~mm}$ length, $2 \mathrm{~mm}$ thickness) were used in the stereognostic tasks.

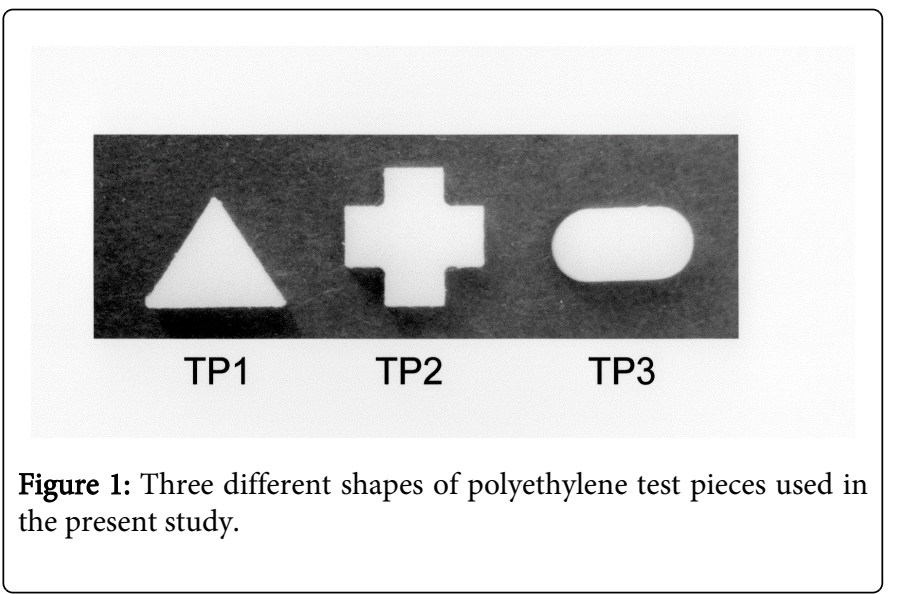

These test pieces were selected from the six test pieces used in the previous report [13] on the basis of the subject's ability to detect the differences in simple shapes. A fine thread (diameter: $0.2 \mathrm{~mm}$ ) was attached to each Test Piece (TP) to prevent choking.

The tasks were performed in a room where faint natural ambient sounds, like the murmur of a stream, were played by tape player to promote a feeling of relaxation. Subjects were fitted with a probe set covering the frontal cortex area, including the prefrontal area (Figure 2 ), and performed all tasks in a sitting position with their eyes closed according to the examiner's instructions.

After resting for $10 \mathrm{sec}$, the subject opened their mouth and extended their tongue. The examiner, who was holding the thread attached to a TP, without touching the TP directly, placed the first TP (TP1) on the center of the tongue dorsum of the subject. The subject moved the TP using their tongue for discrimination without letting it touch their teeth or gums (experimental task 1). After $20 \mathrm{sec}$, TP1 was removed by the examiner and the subject rested for $15 \mathrm{sec}$. The task was repeated using TP2 and TP3 as experimental tasks 2 and 3 (Figure 3). In the control task, a TP was placed on the tongue for $20 \mathrm{sec}$ without moving it.

The levels of oxyhemoglobin (oxy-Hb), deoxyhemoglobin (deoxy$\mathrm{Hb}$ ), and total hemoglobin (total-Hb) were measured using a 45channel, functional near-infrared imaging device (NIRStation OMM-3000; Shimadzu Co., Kyoto, Japan).

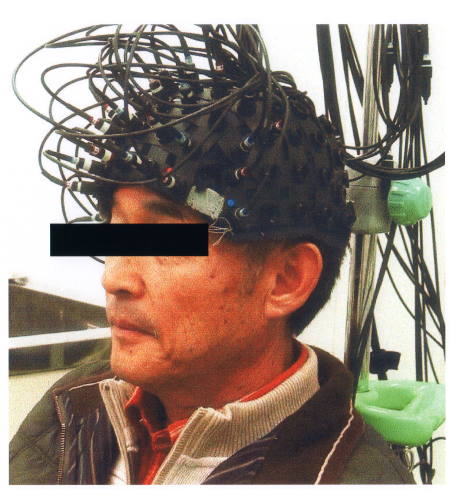

Figure 2: A subject wearing a probe set with a 45 channel functional near-infrared imaging device.

\begin{tabular}{|c|c|c|c|c|c|c|c|c|}
\hline $\begin{array}{c}\text { Rest } \\
10 \mathrm{~s}\end{array}$ & $\begin{array}{c}\text { Task1 } \\
20 \mathrm{~s}\end{array}$ & $\begin{array}{c}\text { Rest } \\
15 \mathrm{~s} \\
\text { (recov- } \\
\text { ery) }\end{array}$ & $\begin{array}{c}\text { Rest } \\
10 \mathrm{~s}\end{array}$ & $\begin{array}{c}\text { Task2 } \\
20 \mathrm{~s}\end{array}$ & $\begin{array}{c}\text { Rest } \\
15 \mathrm{~s} \\
\text { (recov- } \\
\text { ery) }\end{array}$ & $\begin{array}{c}\text { Rest } \\
10 \mathrm{~s}\end{array}$ & $\begin{array}{c}\text { Task3 } \\
20 \mathrm{~s}\end{array}$ & $\begin{array}{c}\text { Rest } \\
15 \mathrm{~s} \\
\text { (recov- } \\
\text { ery) }\end{array}$ \\
\hline
\end{tabular}

Figure 3: Task design. TP1, TP2, and TP3 from Figure 1 were used in task 1 , task 2 , and task 3 , respectively.

\section{Data Analysis}

All volunteers were treated as subjects and subjects were not divided into any groups according to age and gender before measurement of the brain activities which were measured randomly regardless of age and gender. Data from all subjects was used for analysis of results.

The mean value of $\mathrm{Hb}$ levels for the three 10 -sec rest periods prior to experimental tasks 1-3 was subtracted from the mean value of $\mathrm{Hb}$ levels for the experimental tasks 1-3 and used as the experimental $\mathrm{Hb}$ level. The control $\mathrm{Hb}$ level was calculated in the same way. The stereognostic task-attributed change in $\mathrm{Hb}$ levels was calculated by subtracting the control $\mathrm{Hb}$ level from the experimental $\mathrm{Hb}$ level.

For the mapping analysis, values were obtained by subtracting the mean $\mathrm{Hb}$ levels of the three 10 -sec rest periods from the mean $\mathrm{Hb}$ levels calculated at $7 \mathrm{sec}$ post task initiation in tasks 1-3.

\section{Statistical analysis}

A chi-square test was used to examine the association between age groups and $\mathrm{Hb}$ level increase. A $\mathrm{p}$-value of less than 0.05 was considered significant.

\section{Results}

\section{Changes in oxy-Hb levels attributed to the stereognostic task}

The stereognostic task-attributed change in oxy-Hb levels was calculated for every channel to determine the number of channels that showed an increase or decrease in oxy-Hb levels. The results are shown in Table 1. 
Page 3 of 5

\begin{tabular}{|c|c|c|c|c|c|}
\hline $\begin{array}{l}\% \text { of channels (of } 45 \text { ) } \\
\text { showing an increase in } \\
\text { oxy-Hb level }\end{array}$ & $\geq 80 \%$ & $\geq 60 \%$ & $\geq 40 \%$ & $\geq 20 \%$ & $\geq 0 \%$ \\
\hline \multirow{14}{*}{ Number of channels ${ }^{a}$} & $45(0)$ & $35(4)$ & $26(13)$ & $12(16)$ & $8(22)$ \\
\hline & $43(0)$ & $35(9)$ & $25(7)$ & $12(17)$ & $8(28)$ \\
\hline & $43(2)$ & $34(4)$ & $21(7)$ & $11(18)$ & $5(30)$ \\
\hline & $42(0)$ & $30(4)$ & $19(15)$ & $11(23)$ & $0(39)$ \\
\hline & $41(3)$ & $30(8)$ & $19(20)$ & $9(11)$ & \\
\hline & $41(4)$ & $30(11)$ & $18(16)$ & & \\
\hline & $41(4)$ & $30(11)$ & & & \\
\hline & $39(3)$ & $30(11)$ & & & \\
\hline & $39(4)$ & & & & \\
\hline & $38(4)$ & & & & \\
\hline & $38(5)$ & & & & \\
\hline & $36(5)$ & & & & \\
\hline & $36(6)$ & & & & \\
\hline & $36(7)$ & & & & \\
\hline
\end{tabular}

\begin{tabular}{|c|c|c|c|}
\hline \multirow{3}{*}{$\begin{array}{l}\text { Time of life }{ }^{a} \text { of subjects (age range, mean } \\
\text { age, } n \text { ) }\end{array}$} & \multicolumn{3}{|c|}{ Number of subjects } \\
\hline & \multicolumn{3}{|c|}{$\begin{array}{l}\text { Degree of increased oxy-Hb } \\
\text { level }\end{array}$} \\
\hline & High & Moderate & Low \\
\hline $\begin{array}{l}\text { Adolescents }(18-24 \text { years, } 20.8 \pm 1.4 \text { years, } \\
\text { 14) }\end{array}$ & 9 & 5 & 0 \\
\hline $\begin{array}{l}\text { Young adults }(26-44 \text { years, } 34.0 \pm 6.3 \text { years, } \\
\text { 5) }\end{array}$ & 4 & 1 & 0 \\
\hline $\begin{array}{l}\text { Middle-aged ( } 45-63 \text { years, } 55.8 \pm 6.0 \text { years, } \\
\text { 12) }\end{array}$ & 7 & 2 & 3 \\
\hline Seniors ( $\geq 65$ years, $68.7 \pm 4.1$ years, 6 ) & 0 & 1 & 5 \\
\hline
\end{tabular}

Table 2: Relationship between age and degree of increased oxy- $\mathrm{Hb}$ levels at $7 \mathrm{sec}$ post task initiation. ${ }^{\text {a }}$ Time of life classification is based on the document (Kenkou Nippon 21, http://www1. mhlw.go.jp/ topics/kenko21_11/s0.html) by the Ministry of Health, Labour and Welfare, Japan.

In about $65 \%$ of subjects, an increased oxy-Hb level was observed in more than half of measured areas (Table 1), and most subjects showed increased oxy-Hb levels in the frontal cortex, including the prefrontal area (Table 2 and Figure 4).

Table 1: Number of channels showing an increase in oxy-Hb level for each of 37 subjects. ${ }^{\text {a: }}$ Numbers shown in parentheses are the number of channels showing a reduced oxy-Hb level for each of 37 subjects.

There were individual differences in the number and site of channels showing a stereognostic task-attributed change in oxy- $\mathrm{Hb}$ levels. Performance of the stereognostic tasks increased oxy-Hb levels in at least 1 of the 45 channels in 36 of 37 subjects, and 22 of the subjects showed increased oxy-Hb levels in more than $60 \%$ of the channels. On the other hand, oxy-Hb levels decreased in a number of channels in 34 of 37 subjects; however, only 3 subjects showed reduced $\mathrm{Hb}$ levels in more than $60 \%$ of the channels.

\section{Relation between age and stereognostic task-attributed $\mathrm{Hb}$ level increase}

$\mathrm{Hb}$ levels at $7 \mathrm{sec}$ post task initiation were used to map the degree of change in $\mathrm{Hb}$ levels. It was found that the changes in oxy- and total$\mathrm{Hb}$ levels were similar, while the deoxy-Hb levels did not change to the same degree (Figure 4).

There were individual differences with regard to the site and size of the cortical area involved. The subjects were divided based on the degree of oxy-Hb level increase into three groups by our visual evaluation: highly increased oxy-Hb level (Figure 4A), moderately increased oxy-Hb level (Figure 4B), and mildly increased oxy-Hb level (including non-increase, Figure 4C). More than half of the subjects belonged to the highly increased $\mathrm{Hb}$ level group (Table 2).

To investigate whether the $\mathrm{Hb}$ level increase varied with age, subjects were divided by age into four groups as defined by a document provided by the Ministry of Health, Labour and Welfare, Japan. The relation between the oxy-Hb level groups and the age groups was examined, and there was a significant negative correlation between age and degree of oxy-Hb level increase (Table 2, $\mathrm{P}<0.001$ ).

\section{Discussion}

Compared to fMRI, the spatial resolution of fNIRS is reduced; however, fNIRS is easily-transferable and involves minimal cost. The fNIRS technique can be used to noninvasively examine and measure the brain activity in a natural state. Therefore, for the present study, we utilized fNIRS to measure brain blood flow.

In the present study, we examined brain activity, which is visualized by blood flow, by measuring the change in $\mathrm{Hb}$ levels during oral stereognostic tasks using fNIRS. In most subjects, an increase in $\mathrm{Hb}$ levels was observed. For comparison of the degree of $\mathrm{Hb}$ level increase, we mapped the changes in $\mathrm{Hb}$ levels at 4, 7, 10 and $20 \mathrm{sec}$ post task initiation, normalized to the rest level. We used the values at $7 \mathrm{sec}$ for mapping due to the increase in level being the highest. Although little is known about the brain activity attributed to oral stereognosis, Fujii et al. [19] reported oral stereognosis-specific activated brain areas in young adults using fMRI, showing activation in the primary somatosensory area, primary motor area, supramarginal gyrus, premotor area, supplementary motor area, fusiform gyrus, frontopolar area, dorsolateral prefrontal cortex, and insular cortex. Although we did not identify the activated areas in detail as the fMRI study did, the map of areas with an increase in Hb-levels (Figure 4), in addition to the number of channels showing a similar increase (Table 1), indicate that these brain areas are strongly associated with the ability of the tongue to discriminate shapes.

It was reported that aging reduces cerebral blood flow in association cortices during rest, yet the influence of age on functional brain activation is controversial [20-26]. Aging differs among individuals, and the areas affected by aging may differ depending on the task $[23,27,28]$. The fMRI study was performed using only young adults, and the effect of aging was not examined [19]; however, our results suggest that aging affects the oral stereognostic task-attributed change in cerebral blood flow (Table 2). A stereognostic task using the tongue may not effectively stimulate the prefrontal area of elderly individuals 
Citation: Kawagishi S, Toshiko T, Yoshino K, Shimodozono M (2014) Brain Activity during Stereognostic Discrimination Using the Tongue Measured By Functional Near-Infrared Spectroscopy. Aging Sci 2: 128. doi:10.4172/2329-8847.1000128

Page 4 of 5

as much as it does in young adults. We previously showed that the stereognostic ability of the tongue declines with age [12], assessed by identifying twenty test pieces of different shapes placed in the oral cavity. The percentage of correct identification of each test piece in that study was as follows: for TP1, young adults $81.3 \%$, elderly $45.2 \%$; for TP2, young adults $77.0 \%$, elderly $25.7 \%$; for TP3, young adults $70.0 \%$, elderly $27.6 \%$. The reduced stereognostic ability of the tongue in elderly individuals may have been due to the difficulty of increasing the blood flow in the prefrontal area.
Fig $4 \mathrm{~A}$

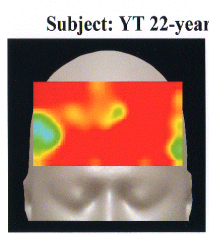

oxy-Hb

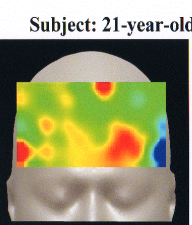

oxy-Hb

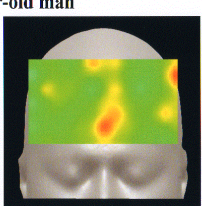

deoxy-Hb

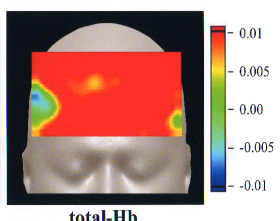

total-Hb

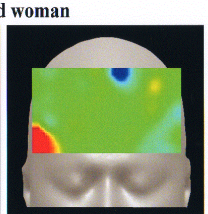

deoxy-Hb

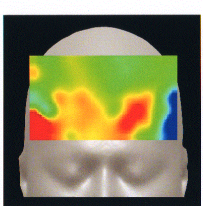

total-Hb
Fig.4B
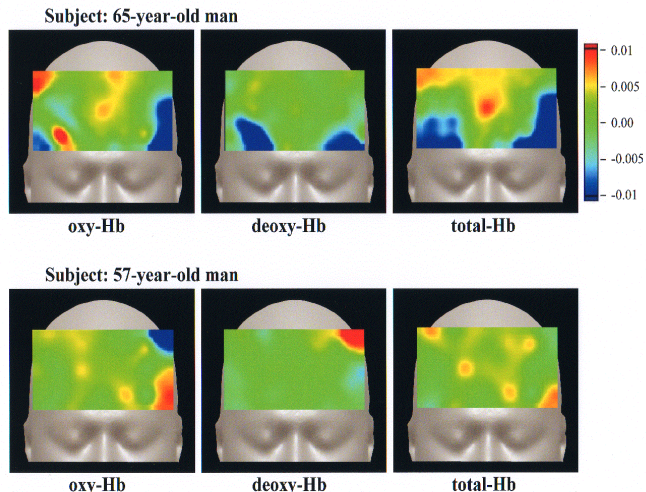

deoxy-Hb

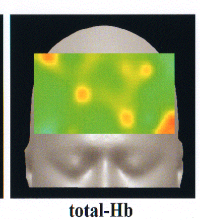

Fig.4C

Subject: 41-year-old man
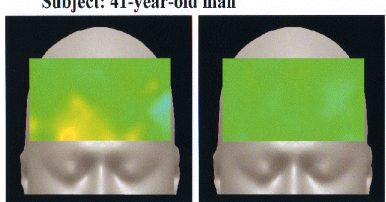

deoxy-Hb

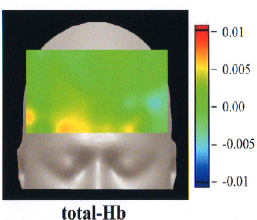

Subject: 75-year-old man

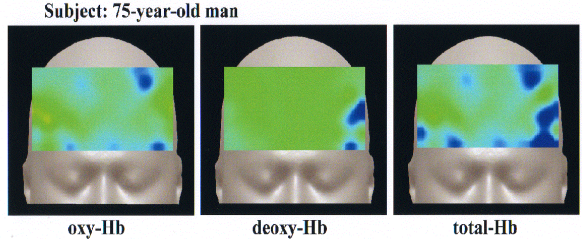

Figure 4: Mapping of the change in $\mathrm{Hb}$ levels at $7 \mathrm{sec}$ post task initiation. 4A, cases with highly increased Hb levels; 4B, cases with moderately increased $\mathrm{Hb}$ levels; $4 \mathrm{C}$, cases with mildly increased $\mathrm{Hb}$ levels.

The MRI study by Fujii et al. [19] did not refer to a large variability in individual differences; however, our results demonstrated large differences in the area and degree of activation among subjects, as well as areas of inactivation. While the exact cause is sure to be complex, individual differences in attention level and the degree of perceived difficulty of the tasks may have contributed to this variability. The present study suggests that one of the factors of this variability is aging. Another possibility is that stereognosis may be involved in several different information processing pathways.

Previously, we revealed that the stereognostic ability of the tongue can be improved in elderly individuals by rehabilitation using a TP [12]. However, it is still unknown how the brain blood flow is improved after this stereognostic activity, and further study is needed to elucidate the mechanisms behind these changes. Using feedback from fNIRS, the knowledge that activation of the brain by sensory information from the oral area may ward off dementia and may motivate rehabilitation of oral functions $[29,30]$.

In conclusion, most subjects showed an increase in oxy-Hb levels in the frontal cortex attributed to the oral stereognostic task; however, the degree and area of these increases showed large individual differences, including cases of $\mathrm{Hb}$ level decrease. In addition, it was demonstrated that the degree of change in oxy-Hb levels may decrease with advancing age.

\section{Acknowledgements}

A part of this study was supported by a Grant-in-Aid for Scientific Research (No. 21500487) from the Japan Society for the Promotion of Science. 
Citation: Kawagishi S, Toshiko T, Yoshino K, Shimodozono M (2014) Brain Activity during Stereognostic Discrimination Using the Tongue Measured By Functional Near-Infrared Spectroscopy. Aging Sci 2: 128. doi:10.4172/2329-8847.1000128

Page 5 of 5

\section{References}

1. Han TR, Paik NJ, Park JW (2001) Quantifying swallowing function after stroke: A functional dysphagia scale based on videofluoroscopic studies. Arch Phys Med Rehabil 82: 677-682.

2. Han TR, Paik NJ, Park JW, Kwon BS (2008) The prediction of persistent dysphagia beyond six months after stroke. Dysphagia 23: 59-64.

3. Nishiwaki K, Tsuji T, Liu M, Hase K, Tanaka N, et al. (2005) Identification of a simple screening tool for dysphagia in patients with stroke using factor analysis of multiple dysphagia variables. J Rehabil Med 37: 247-251.

4. Schmidt RA, Wrisberg CA (2008) Motor Learning and Performance. 4th ed. Human Kinetics Publishers, Champaign, IL.

5. Logemann JA (1998) Evaluation and treatment of swallowing disorders 2nd ed. PRO-ED, Austin, TX.

6. Berry DC, Mahood M (1966) Oral stereognosis and oral ability in relation to prosthetic treatment. Br Dent J 120: 179-185.

7. Grasso JE, Catalanatto FA (1979) The effects of age and full palatal coverage on oral stereognostic ability. J Prosthet Dent 41: 215-219.

8. Müller F, Link I, Fuhr K, Utz KH (1995) Studies on adaptation to complete dentures. Part II: Oral stereognosis and tactile sensibility. J Oral Rehabil 22: 759-767.

9. Jacobs R, Bou Serhal C, van Steenberghe D (1997) The stereognostic ability of natural dentitions versus implant-supported fixed prostheses or overdentures. Clin Oral Investig 1: 89-94.

10. Jacobs R, Bou Serhal C, van Steenberghe D (1998) Oral stereognosis: a review of the literature. Clin Oral Investig 2: 3-10.

11. Boliek CA, Rieger JM, Li SY, Mohamed Z, Kickham J, et al. (2007) Establishing a reliable protocol to measure tongue sensation. J Oral Rehabil 34: 433-441.

12. Kawagishi S, Kou F, Yoshino K, Tanaka T, Masumi S (2009) Decrease in stereognostic ability of the tongue with age. J Oral Rehabil 36: 872-879.

13. Kawagishi S, Tanaka T, Shimodozono M, Yoshino K (2013) Simplifying the assessment of stereognostic ability of the tongue in elderly subjects using six selected test pieces. Aging Sci 1: 111.

14. Shimodozono M, Kawagishi , Yoshino K, Matsumoto S, Kawahira K (2009) Relationship between oral stereognostic ability and videofluoroscopic dysphagia in poststroke patients. The 5th World Congress of the International Society of Physical and Rehabilitation Medicine (ISPRM). June 13-17, Istanbul, Turkey (Abstr. P213).

15. Tamura T, Kanayama T, Yoshida S, Kawasaki T (2002) Analysis of brain activity during clenching by fMRI. J Oral Rehabil 29: 467-472.

16. Tamura T, Kanayama T, Yoshida S, Kawasaki T (2003) Functional magnetic resonance imaging of human jaw movements. J Oral Rehabil 30: 614-622.
17. Takahashi T, Miyamoto T, Terao A, Yokoyama A (2007) Cerebral activation related to the control of mastication during changes in food hardness. Neuroscience 145: 791-794.

18. Takada T, Miyamoto T (2004) A fronto-parietal network for chewing of gum: a study on human subjects with functional magnetic resonance imaging. Neurosci Lett 360: 137-140.

19. Fujii R, Takahashi T, Toyomura A, Miyamoto T, Ueno T, et al. (2011) Comparison of cerebral activation involved in oral and manual stereognosis. J Clin Neurosci 18: 1520-1523.

20. Schroeter ML, Zysset S, Kruggel F, von Cramon DY (2003) Age dependency of the hemodynamic response as measured by functional near-infrared spectroscopy. Neuroimage 19: 555-564.

21. Schroeter ML, Schmiedel O, von Cramon DY (2004) Spontaneous lowfrequency oscillations decline in the aging brain. J Cereb Blood Flow Metab 24: 1183-1191.

22. Ramsøy TZ, Liptrot MG, Skimminge A, Lund TE, Sidaros K, et al. (2012) Healthy aging attenuates task-related specialization in the human medial temporal lobe. Neurobiol Aging 33: 1874-1889.

23. Gauthier CJ, Madjar C, Desjardins-Crépeau L, Bellec P, Bherer L, et al. (2013) Age dependency of hemodynamic response characteristics in human functional magnetic resonance imaging. Neurobiol Aging 34: 1469-85.

24. Haller S, Rodriguez C, Moser D, Toma S, Hofmeister J, et al. (2013) Acute caffeine administration impact on working memory-related brain activation and functional connectivity in the elderly: a BOLD and perfusion MRI study. Neuroscience 250: 364-71.

25. Fabiani M, Gordon BA, Maclin EL, Pearson MA, Brumback-Peltz CR, et al. (2014) Neurovascular coupling in normal aging: a combined optical, ERP and fMRI study. Neuroimage 85 Pt 1: 592-607.

26. Vermeij A, Meel-van den Abeelen AS, Kessels RP, van Beek AH Claassen JA (2014) Very-low-frequency oscillations of cerebral hemodynamics and blood pressure are affected by aging and cognitive load. Neuroimage 85 Pt 1: 608-615.

27. Dushanova J, Christov M2 (2014) The effect of aging on EEG brain oscillations related to sensory and sensorimotor functions. Adv Med Sci 59: 61-67.

28. Berti V, Mosconi L, Pupi A (2014) Brain: normal variations and benign findings in fluorodeoxyglucose-PET/computed tomography imaging. PET Clin 9: 129-140.

29. Wu B, Plassman BL, Liang J, Wei L (2007) Cognitive function and dental care utilization among community-dwelling older adults. Am J Public Health 97: 2216-2221.

30. Heuninckx S, Wenderoth N, Debaere F, Peeters R, Swinnen SP (2005) Neural basis of aging: the penetration of cognition into action control. J Neurosci 25: 6787-6796. 